Michele De Beni*

ORCID: 0000-0001-9440-4804

Loppiano, Italy

\title{
Un Maestro come modello di vita e di pensiero
}

\section{The Teacher as a Model of Life and Thought}

\begin{abstract}
Summary: The present article focuses on pedagogical aspects emerging from Chiara Lubich's written work and oral conversations. Here, three major points are elaborated: the importance of the relationship as a fundamental element of education; the importance of the educational model, conceptualised as the need of an Ideal Teacher source of life inspiration; the concept of thought and knowledge as a service of love to the world. Points of conversation were fostered within the framework of these aspects to develop a model upon which education, thought and action are built as means of fraternal service.
\end{abstract}

Keywords: educator; model; knowledge; thought; service.

Sommario: L'articolo intende metter a fuoco aspetti pedagogici che si possono individuare in alcune conversazioni e scritti di Chiara Lubich. Tra questi vengo-

* Michele De Beni, pedagogista, psicoterapeuta, professore di Pedagogia della Persona e della Comunità presso l'Istituto Universitario Sophia di Loppiano (Firenze), docente presso il Centro Studi Interculturali dell'Università di Verona. Coordinatore per l'Italia del progetto internazionale Co.R.T. (Cort Thinking) diretto da Edward de Bono. Contatto: Istrituto Universitario Sophia (www.sophiauniversity.org/it) Via San Vito n. 28, Loppiano, 50064 Figline e Incisa Valdarno (FI) Italia; e-mail: debeni.m@gmail.com 
no qui discusse tre fondamentali questioni riguardanti la primarietà della relazione come elemento costitutivo dell'educazione stessa; l'importanza del modello educativo, inteso come necessità di un Maestro Ideale, ispiratore di vita; il senso del conoscere e del pensare come servizio, per amore del mondo. Nel quadro delle questioni presentate si cerca di suscitare alcuni orizzonti di discussione per un modello fondante il senso dell'educare, del pensare e dell'agire come servizio fraterno.

Parole chiavi: educatore, modello, conoscere, pensare, servire.

Avvicinarsi a figure così nobili e coraggiose come Chiara Lubich, anche solo per metterne in luce alcuni punti del carisma applicato a tematiche educative, è prima di tutto un privilegio, ma anche un'ardua sfida sul piano della ricerca. Dal punto di vista pedagogico, è occasione per riflettere sulla straordinaria attualità della sua testimonianza di vita e di pensiero, nell'ottica prima di tutto di un invito a riprendere la quotidiana, sempre nuova, sfida dell'educare. Di quest'altissima testimone, pur sinteticamente, intendo metter a fuoco alcune questioni fondanti come quella assegnata alla figura del Maestro, alla conoscenza e alla capacità di pensare, richiamando l'eco che ha suscitato in me la lettura di alcuni suoi scritti.

\section{Educazione come vocazione dell'amore}

Partirei da una premessa fondamentale per la fondazione di un discorso pedagogico: Chiara Lubich era specchio fedele delle sue parole e convinzioni. È questo un punto nodale, qualificante che ci deve stare a cuore prima di ogni altra questione, fondamento di tutto il discorso e di tutta la pratica educativa. Infatti, come ha sottolineato Paolo VI, "l'uomo contemporaneo ascolta più volentieri i testimoni che i maestri [...] o se ascolta i maestri lo fa perché sono dei testimoni"'. Teoria-Pratica, Pensiero-Azione, Parola-Vita. È questa l'estrema testimonianza che i giovani si aspettano dagli adulti, richiamati a saldare le parole all'esperienza, gli ideali all'impegno concreto e quotidiano. Solo così un educatore potrà esser autentico, sviluppare le potenzialità dell'educando ed orientarle al bene.

${ }^{1}$ Paolo VI, Evangelii Nuntiandi (Vatican: Libreria Editrice Vaticana, 1975), 41. 
La stessa Lubich in una conversazione del 1971 agli abitanti della Cittadella di Loppiano ${ }^{2}$ riprende il tema della testimonianza, collegandolo a quello della vocazione nella sua duplice valenza: da una parte di "chiamata", "scelta", "passione", "attrazione" verso un Ideale acceso nel cuore da Dio, dall'altra come "dedizione", "sentimento" verso i fratelli. Un'ineffabile sensibilità, che la Lubich aveva sperimentato già come giovane insegnante ${ }^{3}$, spinta da una grande passione sociale ed educativa, capace di dar ragione alla speranza di una vita buona ${ }^{4}$, alla scuola di quel Maestro interiore che l'aveva chiamata a donarsi tutta a lui nell'amore ai fratelli, quindi anche ai più piccoli ed emarginati, non-amati, "di tutte quelle realtà-esperienze umane e sociali per le quali c'è - più che in ogni altra - un urgente e speciale bisogno di educazione"s.

Ed è qui, a mio parere, che si apre un interessante e attualissimo squarcio su quello che ritengo il punto più qualificante dell'educazione come la sentiva e la viveva Chiara Lubich: il suo continuo richiamo alla "primarietà della relazione": cura dei rapporti tra persone, per capirne le motivazioni e le attese, le paure e le contraddizioni, le aspirazioni, per arrivare al cuore e aprire le menti. Una lezione che oggi faremmo bene a riprendere dalle fondamenta per una "scuola veramente educante", persone e comunità non alla mercè dell'efficientismo, ma in cui i legami costituiscono la trama essenziale delle relazioni interpersonali e sociali per una "città ideale", che dà senso e unitarietà alla vita. Da questo punto di vista, la crisi radicale che investe oggi l'educazione non è tanto metodologica, ma assiologia. Non sarebbe, cioè, una crisi di strumenti ma di contenuti valoriali. Essa non riguarda tanto il "come", ma il "perché", la scelta e la giustificazione dei fini da proporre.

\section{Un Maestro come modello}

Nell'ottica di Lubich, l'educazione può esser vista direttamente correlata a quella di testimonianza della propria vocazione, non intesa come semplice atto comunicativo e relazionale ma di amore, dono liberamente offerto, su imitazione del modello educativo di Gesù. Si inizia, così, a introdurre

2 Chiara Lubich, "Gesù Maestro", Nuova Umanità 180 (2008): 607-612.

3 Quando la scuola educa, ed. Michele De Beni (Roma: Città Nuova, 2017).

${ }^{4}$ Conferenza Episcopale Italiana, Orientamenti Pastorali. Educare alla vita buona del Vangelo (Milano: Edizioni S. Paolo, 2010), 29.

5 Chiara Lubich, "Lezione per la laurea honoris causa in Pedagogia", Nuova Umanità 135-136 (2001): 341-352. 
il concetto della necessità di un modello pedagogico trascendentale, metaeducativo, non astratto, ma plasticamente rappresentato dalla figura di un Maestro-testimone di quella vita che, osservata e fatta propria dall'educando, diventa motivo ispiratore dei suoi atteggiamenti. Si potrebbe qui richiamare tutta la tradizione pedagogica cristiana pre-medievale che fa riferimento ad una Pedagogia divina, al "pedagogo" per eccellenza, Dio, che indica il cammino verso la Verità. Da questo punto di vista, il livello divino si innesta in quello umano e lo anima ${ }^{6}$. Realtà e Utopia, pratica e ideale, nella Lubich assumono il carattere unitario di esperienza di vita, centrata sulla figura di Gesù-Maestro-Modello. Non un'astratta adesione ad un modello tramandato dalla Chiesa, ma basata prima di tutto sulla propria esperienza di incontro, autentico e vivificante, storicamente situato, con la Persona di Gesù, presenza viva tra "due o tre riuniti" nel suo nome (Mt 18, 20). Egli, in questo senso, è vero Maestro ${ }^{7}$ perché con i suoi insegnamenti conduce, sostiene ed orienta. Nello stesso tempo è Modello, in quanto, sulla base fenomenologica ed esperienziale dei propri vissuti e di quelli delle persone che hanno seguito questa pratica di vita, Chiara e le sue prime compagne possono affermare, perché lo hanno constatato ogni giorno, quanto straordinari siano gli effetti "salvifici" di questo Modello per l'umanità, nella vita personale, familiare, sociale, economica, culturale. Alla luce di questo Modello, l'educazione non va interpretata come semplice verità rivelata, ma affrontata contemporaneamente secondo una rigorosa analisi pedagogica, che richiede un'adeguata ricerca e spiegazione sul piano razionale ${ }^{8}$. La traccia di questa verità è la prossimità di Dio nel volto degli altri, del fratello. In questo senso il volto dell'altro è prova dell'idea d'Infinito che c'è in ogni essere umano, e, quindi, della più o meno conscia relazione che ciascun uomo ha con Dio. Essa si attualizza,

${ }^{6}$ Clemente Alessandrino, Il Pedagogo (Roma: Città Nuova Editrice, 2005), VII, 54, 1.

${ }^{7}$ Qui il termine "vero Maestro" potrebbe esser messo in contrapposizione all'ubriacatura antropocentrica del post-moderno, per cui occorre essere disponibili a linguaggi che non siano solo quelli della filosofia pratica, ma che si aprano a nuove altezze, alla "rivelazione" gratuita che viene dal di fuori - dall'altro da sé - , alla dimensione trascendente della vita. "Vero Maestro" potrebbe anche significare "valore", cioè degno di esser considerato importante, stimabile, secondo alcune tipiche caratteristiche: come valore in sé, portatore di ideali; come realtà valida per la vita personale e sociale; come scelta soggettiva, che implica una responsabile azione di compromissione; come bene culturale, quadro di riferimento di un popolo, della cultura.

${ }^{8}$ Cf. il titolo originario del Proslogion (1077) di Anselmo d'Aosta: Fides quarens intellectum; per un inquadramento dei problemi educativi trattati dai Padri della Chiesa e dai grandi teologi medioevali [cf. Giuseppe Groppo, “Teologia dell'educazione”, in: Enciclopedia pedagogica, vol. VI (Brescia: La Scuola, 1994), 11787-11798]. 
appunto, nella concretezza della relazione con l'altro. L'altro, perciò, è via per arrivare all'Altro, Infinito fondativo, fine ultimo, ma anche mezzo della relazione stessa tra gli uomini ${ }^{9}$.

\section{Un Maestro di vita e di pensiero}

Se la figura di Gesù-Maestro come modello assume certamente una rilevanza centrale per un'analisi pedagogica degli scritti e conversazioni di Lubich, penso che un ulteriore elemento fondamentale possa esser riconducibile al significato e al valore che Chiara assegna alla conoscenza e al pensiero. Una semplice ma efficace risposta si può rintracciare direttamente dalle stesse testimonianze dei suoi allievi di scuola elementare ${ }^{10}$. Essi in una recente intervista riferiscono che una delle domande più impegnative e ricorrenti della loro insegnante, di fronte a un problema, a un dubbio o, semplicemente, per far emergere e capire un punto di vista diverso, era la domanda diretta ed esplicita: "Ma, tu, cosa ne pensi?", invito provocatorio per stimolare un dialogo, per aiutare l'altro a interrogarsi, a chiarire, a porre ipotesi di soluzione, e così ad assumersi la responsabilità di un pensiero proprio. Un giovane, uno studente, infatti, sarà tanto più formato e competente quanto più sollecitato a una riflessione sistematica riguardante non solo il modo in cui apprende ma anche come affronta ogni aspetto della sua vita personale, sociale e culturale.

"Ma, tu, cosa ne pensi?" potrebbe essere, quindi, il motivo-guida di un metodo orientato a una più diretta responsabilizzazione dello studente soprattutto sul piano della ricerca, personale e culturale. L'educatore, in questo senso, non si pone in cattedra per ammaestrare, nel senso di indottrinare, ma per fornire una sua "versione", punto di vista. Il suo compito non è di coprire o di uniformare, ma invito a intraprendere un cammino, e di ricondurre all'unità i diversi punti di vista ${ }^{11}$; stimolando, quindi, le potenzialità e gli atteggiamenti partecipativi e creativi degli allievi, coinvolti nell'atto del conoscere e del pensare, visto nella sua essenza di processo co-educativo. Maestro come modello ideale - a cui ispirare l'azione educativa - ed eserci-

9 Emmanuel Lévinas, Di Dio che viene all'idea (Milano: Jaca Book, 1997).

${ }^{10}$ Donato Chiampi, "La maestra Silvia non aveva la matita rossa", in: Essere educatori, coraggio di una presenza, ed. Michele De Beni, dvd-intervista ai suoi ex alunni (Roma: Città Nuova, 2014).

${ }^{11}$ Cf. Maria Zambrano, Chiari nel bosco (Milano: Feltrinelli, 1991). 
zio del pensiero come azione pratica e speculativa possono quindi esser rappresentati come un continuum, in cui Ideale e Vita si richiamano a vicenda.

\section{Conoscere e Pensare per amore}

Potremmo interrogarci perciò in modo più specifico su quale sia il significato e il ruolo assegnato dalla Lubich alla conoscenza, al pensare e alla loro relazione con un Modello pedagogico, che per Chiara Lubich si identifica con la Persona di Gesù. A questo riguardo, nella conversazione ai cittadini della Cittadella di Loppiano, la Lubich afferma che:

se Loppiano è una scuola, se ha la funzione della scuola, essa, però, è una scuola tutta particolare e molto originale. Non sono infatti i libri, le aule, gli studi, le prime cose che la fanno scuola. No, Loppiano è una scuola perché qui vi è un Maestro. Egli è colui che ha ispirato ai suoi fondatori come andava concepita questa città, come andava iniziata, come andava portata avanti. Egli è anche colui che abita tra gli abitanti di questa città. Noi sappiamo che chi ha ispirato questa città e colui che abita tra gli abitanti di essa, è Gesù ${ }^{12}$.

Socrate, in lotta con il modo decadente tipico del suo tempo, si riferisce al pensiero come testimonianza della verità e come rimedio (pharmacon) contro i mali della vita. Solo la divinità è sapiente, mentre il sapere umano vale poco o nulla, per cui chi ritiene di possedere il sapere, e pretende il sapere assoluto, in realtà tradisce la vera conoscenza. L'uomo, quindi, deve prima di tutto saper riconoscere la propria finitezza. Ma se il sapere come "sapere per essere" parte dal riconoscimento della propria finitezza, qual è il suo fondamento, il senso vero, la verità verso cui tendere? ${ }^{13}$

12 Lubich, “Gesù Maestro”, 607-612.

13 Come afferma Edgar Morin, è sul senso di finitezza e di limite che si basa anche la comprensione, quale etica della comprensione, "orientata alla tolleranza. Infatti se sappiamo comprendere prima di condannare, saremo sulla via dell'umanizzazione delle relazioni umane" [Edgar Morin, I setti saperi necessari all'uomo futuro (Milano: Cortina, 2001), 104]. Un significativo riferimento al senso del limite umano è rintracciabile nella tradizione monastica, in cui il "pregare" si configura non solo come adorazione ma come "domanda", un chiedere alla verità di manifestarsi. "Nell'abbandono disponibile alla rivelazione dell'Assoluto, il monaco traccia un sentiero del pensare" che non è un adeguamento alla correttezza logico-sintattica ma al significato autentico della verità [Carlo Scilironi, In cammino verso l'uomo (Milano: S. Paolo, 1994), 24]. 
Un modello di risposta ci viene sicuramente dal testo "Gesù Maestro"14 di Chiara Lubich. Il discorso qui assume una sua caratteristica struttura argomentativa: non cerca tanto di descrivere la realtà, quanto di comprenderla nella sua ragione fondativa. Si propone, cioè, di pensare non solo alla semplice realtà ma a tutta la realtà, applicando all'osservazione quel "principio di trascendenza" che permetta di andare oltre il "principio di evidenza"15. Il pensare, quindi, qui non è inteso solo come un'opzione logico-scientifica di verità, ma piuttosto come certezza dello spirito, e quindi come comprensione interiore. Essa non esclude affatto la ricerca e la spiegazione dei nessi causali, ma ne giustifica il significato entro un quadro di valore.

Occorre precisare, quindi, che l'opzione così decisamente espressa verso un modello trascendente di conoscenza, espresso per i cristiani dalla vita e dall'insegnamento di Gesù, non è da intendere come un'essenza definita a priori, ma vera e propria opera di ricerca, di intima connessione tra una pedagogia dell'esistenza (con) e una pedagogia dell'essenza ${ }^{16}$. Da questo punto di vista, Gesù, quale uomo-Dio, riassume le caratteristiche della realtà e dell'utopia, della temporalità e della trascendenza ${ }^{17}$. Infatti, quando Chiara afferma - riferendosi alla città come scuola di vita - che essa è una scuola tutta originale dove non sono i libri, le aule, gli studi, le scuole che la fanno scuola, ma è la presenza di un Maestro, di "Gesù, con le sue lezioni molto particolari, che non hanno niente a che fare neanche con i più grandi maestri della terra" ${ }^{18}$, ella non intende svalutare l'insegnamento né lo studio. Infatti, sia l'uomo sia Gesù sono maestri, anche se in forme e a livelli diversi: l'uomo insegna verità contingenti, suffragate dalla ragione, mentre Gesù-Maestro insegna verità universali, che parlano alla coscienza. E ciò potrebbe apparire agli uomini, anche ai più saggi - prosegue la Lubich - come una "scienza che

${ }^{14}$ Lubich, "Gesù Maestro", 607-612.

15 Edmund Husserl denunciava la pericolosa perdita di senso della scienza moderna. Essa è tanto più grave se rapportata al campo educativo, in cui dovrebbe costituire la finalità prioritaria [cf. Edmund Husserl, L'idea della fenomenologia (Bari: Laterza, 1992), 64; cf. anche Carlo Nanni, L'educazione tra crisi e ricerca di senso (Roma: Libreria Ateneo Salesiano, 1986)].

${ }^{16}$ Cf. Bodgan Suchodolski, Pedagogia dell'essenza e pedagogia dell'esistenza (Roma: Armando, 1965); Maurice Debesse, Gaston Mialaret, Trattato delle scienze pedagogiche, vol. 1 (Roma: Armando, 1971), 99.

${ }^{17}$ Alla luce di questo Modello-Orientamento di pensiero, l'educazione non va interpretata come semplice verità rivelata, ma affrontata contemporaneamente secondo una rigorosa analisi, che richiede un'adeguata ricerca e spiegazione sul piano razionale [cfr. Anselmo d'Aosta, Proslogion (Milano: Rizzoli BUR, 2012), 1, 27 (1077)].

18 Lubich, "Gesù Maestro", 607-612. 
è stoltezza", cioè "non ragionevolezza", in quanto non centrata esclusivamente sulla ragione. Ma è proprio questo il passaggio più significativo del discorso di Chiara: che qui si apre ad una scienza-sapienza ${ }^{19}$ non contraria alle dimostrazione, né all'indagine psicologica e sociale, ma che le ingloba e, nello stesso tempo, le trascende come "scienza della coscienza". L'orizzonte si allarga e si innalza oltre, al di là del confine che il pensiero postmoderno, tutto autocentrato, sembra incapace di superare. L'invito di Gesù che Chiara comprende come chiamata a "lasciare i maestri" e a seguire i Suoi insegnamenti ${ }^{20}$, può esser inteso come ripresa del tema prima introdotto del rapporto tra scienza e sapienza. La ricerca di una fonte primaria di senso diventa il motivo dominante, una questione di scelta gerarchica in cui il significato viene prima dell'oggetto stesso di conoscenza. È Gesù che dà forma a tutti gli altri significati. Egli è il Senso Infinito che raccoglie i diversi significati finiti. Da questo punto di vista il "sacrificio di tutte le verità che gli uomini possono darci" non richiede la loro rinuncia, né il rifiuto della necessaria autonomia della ricerca scientifica, con obiettivi e metodi propri. C'è piuttosto una ricontestualizzazione di senso che non si trova nella scienza in quanto tale ${ }^{21}$.

Si esplicita una delle condizioni pedagogiche essenziali, cioè la sua dimensione teleologica, non vista nella sua astrattezza formale ma assunta nella sua tipica dialettica, intesa nel senso cristiano, tra uomo-mondo e uomo-virtus. Non più, quindi, come nella tradizione aristotelica, assunzione di finalità etico-politiche o umanistico-retoriche, ma di fondamenti di natura trascendente, il cui contenuto essenziale è la Parola di Dio. Sta qui il preannuncio, anche, di un concetto pedagogico molto elevato, il cui ethos è espresso dal comandamento nuovo dell'amore reciproco $(\mathrm{Gv} 13,34)$, garantito - come

${ }^{19} \mathrm{Ci}$ si riferisce qui al noto rapporto e distinzione tra saggezza e intelligenza. Come affermano anche gli studiosi più rappresentativi delle scienze cognitive, "occorre occuparsi del pensiero che porta alla saggezza piuttosto di quello che determina l'intelligenza. In fondo, se si diventa saggi non è così difficile poi diventare anche intelligenti. Se si comincia invece dall'esser intelligenti si hanno poche speranze di diventare saggi, perché è facilissimo cadere nella «trappola» dell'intelligenza" [Edward De Bono, Io ho ragione, tu hai torto (Milano: Sperling e Kupfer, 1990)].

${ }^{20}$ È qui evidente la profonda affinità spirituale tra Chiara Lubich e S. Agostino, e quella più pedagogica con Clemente Alessandrino, entrambi affascinati dalla figura di Cristo, Signore e Pedagogo dell'Umanità. Così anche negli scritti di Romano Guardini sulla Pedagogia cristiana [Romano Guardini, Persona e libertà (Brescia: La Scuola, 1987), 24].

${ }^{21}$ Anche il paradigma ecologico oggi ha elaborato una chiara consapevolezza del limite del metodo oggettivo [cf. Luigina Mortari, Epistemologia della ricerca pedagogica (Verona: Universitaria, 2003), 31-36]. 
ricorda Chiara - dalla presenza di Colui che abita, suscita la vita, tra gli abitanti di una famiglia, di una scuola, di una città, di una comunità e della cultura più vasta.

\section{Pensare-Servire}

Trova spiegazione così l'invito di Gesù: "E non fatevi chiamare 'maestri' perché uno solo è il vostro Maestro, il Cristo" (Mt 23, 10). Gesù, è ovvio, non intende affermare che la comunità non abbia bisogno di maestri, ma soprattutto di testimoni di vita, orientati verso Lui, che è Amore. Un pensare è vero, quindi, se non si ripiega su se stesso ma sa farsi pensiero esodale, se da monologo sa farsi racconto: di sé a se stessi e di sé agli altri; e se sa farsi dialogo: con il proprio mondo interiore e con il mondo sociale e culturale.

È così che si dà vita ad una comunità di ricerca, ben diversa da quello che si intende oggi per "comunità di scienziati"22. Essa è più che altro una comunità "in ricerca": come tale non rinuncia certamente alle rigorose leggi della razionalità (pensiero critico), allo sviluppo della scoperta e dell'immaginazione (pensiero creativo), ma fa leva sul senso di appartenenza e di condivisione, che lega i suoi membri tra loro, sul pensiero espresso nella cura come servizio. Infatti, come conclude Gesù nel brano di Matteo $(23,11)$ : "Chi è il più grande tra voi sia vostro servo", Gesù invita ad usare il pensiero non come potere dell'uomo sull'uomo, ma come via di responsabilità reciproca. Chiede di rinunciare al potere, non come rinuncia a pensare, ma come scelta del non-potere, cioè di servire ${ }^{23}$.

Anche il pensare, allora, se "per amore", può diventare un autentico atto di servizio alla persona, all'umanità. Da questo principio dell'amore scambievole scaturisce appunto sia l'atteggiamento autorevole e democratico dell'educatore, come processo attivo e compartecipato, sia il concetto di formazione continua che coinvolge educando e educatore, pur secondo ritmi e piani diversi, in un processo circolare, di scambio e di tensione comuni verso il "dover essere". È qui che l'educatore vive la duplice dimensione del maestro e dell'allievo: non una dicotomia, ma un tutto unitario che, da una parte si deve confrontare con l'azione educativa quotidiana e la persona dell'educando, dall'altra con la ricerca di senso e con le finalità della vita.

\footnotetext{
${ }^{22}$ Matthew Lipman, Educare al pensiero (Milano: Vita e Pensiero, 2000).

${ }^{23}$ Come ben si esprime Carlo Scilironi, "potere del non-potere è il servizio" [Scilironi, In cammino, 115].
} 
Gesù, quindi, non ha costruito una dottrina, cioè una teoria, ma piuttosto ha testimoniato con la Sua vita le verità di cui parlava e in cui credeva. Da qui il Suo insegnamento, che potrebbe disorientare se paragonato alla struttura assunta dal linguaggio e dai metodi della ricerca razionale. In questo senso, esso potrebbe apparire degno di poca considerazione perché non scientifico ma, da un punto di vista fenomenologico, non possiamo sostenere che l'esperienza sia in contrasto con la scienza. Anzi, essa, per certi versi ne anticipa i risultati, in quanto esistenza ed essenza sono compresenti. Infatti l'esistenza costituisce il concreto determinarsi dell'essenza. La vita di Gesù, che ne costituisce anche l'insegnamento, non è per nulla in contrasto con la possibilità di tradurla in prassi educativa e, quindi, empiricamente da studiare e metter alla prova dei fatti e dei risultati. Se ne può, allora, ipotizzare un Modello che, sulla base di riflessioni a livello teologico, possa esser ragionevolmente dimostrato e compatibile sia dal punto di vista culturale che scientifico.

Si può affermare, quindi, che se Gesù-Maestro può esser letto sotto il profilo di modello teologico ed esperienziale, dall'altra esso può dar il via a nuove e suggestive ipotesi di ricerca pedagogica che concorrano a costituire una teoria anche sul piano della conoscenza.

\section{Pensare-Essere Nulla per Amore}

Possiamo chiederci allora come il pensiero possa tradursi in atto d'amore. È una dinamica che unisce vita e pensiero, teoria e prassi, ricerca personale e dialogo. È un processo di ascolto, di dono e di comunione, di un pensare che è compartecipazione, introduzione, attraverso il Figlio-Gesù, nello Spazio e nella Logica della Trinità ${ }^{24}$. In questa Logica, Gesù sulla croce che grida "Dio mio, Dio mio, perché mi hai abbandonato?" (Mt 27, 46) è il vertice dell'amore, "il punto culmine, la più bella espressione dell'amore"25, in quan-

${ }^{24}$ Sul concetto di Trinità, vista attraverso l'ottica di Gesù-Abbandonato, Piero Coda scrive: "Dio è Sé (l’Essere) «divenendo», in Sé, l'Altro da Sé: morendo a Sé, come Padre, «essendo» (generando) il Figlio, e «ritrovando Sé» nella Koinonia-libertà che è lo Spirito. L'Essere-Dio di Dio è dunque tutto e solo nel dinamismo della sua vita «inter-personale», in cui ciascuno dei Tre è Dio, l'Uno, in quanto «non è», in quanto «si dà», in quanto dà ciò che è più suo, l'Esser-Dio" [La Trinità e il pensare, edd. Piero Coda, Andreas Tapken (Roma: Città Nuova, 1997), 15].

${ }^{25}$ Chiara Lubich, "Mistero d'amore", Gen 18 (1984): 3. 
to "miracolo dell'annullamento di ciò che è". Il pensiero fecondo, quindi, è tale se è "vuoto di sé" 26 . Il nulla, allora, non è qui inteso in antitesi all'essere, ma l'essere stesso vissuto come amore. Infatti, nelle varie 'lezioni' di Chiara Lubich nulla, dono e amore sono termini strettamente correlati, che in "Gesù Abbandonato" trovano la loro più alta espressione, emblema dell'estremo amore: di Gesù, il Tutto, che si fece Nulla per amore ${ }^{27}$.

È la stessa logica che dovrebbe muovere il pensiero che si permea d'amore, si fa uno con l'amore. In tal modo - per amore - io perdo il mio pensiero, lo sposto, lo faccio tacere per accogliere la ricchezza del pensiero e della totalità che è nell'altro. Solo un cambiamento di questo tipo può portare ad una sintesi costruttiva, dinamica, capace di superare le frammentazioni e accogliere la molteplicità dei saperi ${ }^{28}$ : obiettivo non certo facile e immediato, ma di cui un vero educatore deve saper assumersi personalmente e collettivamente la responsabilità, per ritrovare insieme il coraggio dell'educare, di schiudere i nostri occhi, quelli dei nostri giovani, verso spazi nuovi, di vera intelligenza. Qui decisamente si introduce l'elemento essenziale che riguarda il "metodo" attraverso cui l'educando può percorrere la strada della propria piena realizzazione: 1'amore al fratello. Effetto di questo metodo è la pienezza della gioia, cioè la felicità, che richiede di saper vivere 1' "atteggiamento di vedere Gesù gli uni negli altri" ${ }^{29}$, presenza incarnata di quel Maestro, vincolo di unità tra persone (quindi anche tra educatore-educando, tra educandi, tra educatori) legate da un patto di amore reciproco. È in quest'intreccio di motivi ideali e di esperienza che l'educazione diventa possibile, non un'utopia astratta, ma incontro, giorno per giorno coraggiosamente cercato, "Cielo stellato sopra di me e legge morale in me" ${ }^{\text {"30 }}$.

${ }^{26}$ Chiara Lubich, "Conversazione ai focolarini (8.12.1971)" - cit. di. in: Judith Povilus, Gesù in mezzo nel pensiero di Chiara Lubich (Roma: Città Nuova, 1981), 83.

${ }^{27}$ Per un approfondimento del concetto di "nulla" e di "farsi nulla per amore" si confronti il saggio di Hubertus Blaumeiser, "Un mediatore che è Nulla", Nuova Umanità 117-118 (1998): 385-407.

${ }^{28}$ Piero Coda, "Alcune riflessioni sul conoscere teologico", Nuova Umanità 122 (1999): 191-206.

29 Sta qui anche la ragione d'essere dell'educazione, che non può prescindere dalla dimensione etica, storicamente e teleologicamente fondata [cf. Alasdair MacIntyre, Dopo la virtù. Saggio di teoria morale (Milano: Feltrinelli, 1988)].

${ }^{30}$ Immanuel Kant, Critica della ragion pratica (Bari: Laterza, 1974), 197. 


\section{Bibliografia}

Anselmo d'Aosta. Proslogion. Milano: Rizzoli BUR, 2012.

Blaumeiser, Hubertus. "Un mediatore che è Nulla". Nuova Umanità 117-118 (1998): 385-407.

Chiampi, Donato. "La maestra Silvia non aveva la matita rossa". In: Essere educatori, coraggio di una presenza, ed. Michele De Beni, dvd-intervista ai suoi ex alunni. Roma: Città Nuova, 2014.

Clemente Alessandrino. Il Pedagogo. Roma: Città Nuova Editrice, 2005.

Coda, Piero. "Alcune riflessioni sul conoscere teologico". Nuova Umanità 122 (1999): 191-206.

Conferenza Episcopale Italiana. Orientamenti Pastorali. Educare alla vita buona del Vangelo. Milano: S. Paolo, 2010.

Debesse, Maurice, Gaston Mialaret. Trattato delle scienze pedagogiche, vol. 1. Roma: Armando, 1971.

De Bono, Edward. Io ho ragione, tu hai torto. Milano: Sperling e Kupfer, 1990.

Groppo, Giuseppe. “Teologia dell'educazione”. In: Enciclopedia pedagogica, vol. VI, 11787-11798. Brescia: La Scuola, 1994.

Guardini, Romano. Persona e libertà. Brescia: La Scuola, 1987.

Husserl, Edmund. L'idea della fenomenologia. Bari: Laterza, 1992.

Kant, Immanuel. Critica della ragion pratica. Bari: Laterza, 1974.

La Trinità e il pensare, edd. Piero Coda, Andreas Tapken. Roma: Città Nuova, 1997.

Lévinas, Emmanuel. Di Dio che viene all'idea. Milano: Jaca Book, 1997.

Lipman, Matthew. Educare al pensiero. Milano: Vita e Pensiero, 2000.

Lubich, Chiara. "Lezione per la laurea honoris causa in Pedagogia". Nuova Umanità 135-136 (2001): 341-352.

Lubich, Chiara. "Mistero d'amore". Gen 18 (1984): 3.

Lubich, Chiara. "Gesù Maestro". Nuova Umanità 180 (2008): 607-612.

MacIntyre, Alasdair. Dopo la virtù. Saggio di teoria morale. Milano: Feltrinelli, 1988.

Morin, Edgar. I setti saperi necessari all'uomo futuro. Milano: Cortina, 2001.

Mortari, Luigina. Epistemologia della ricerca pedagogica. Verona: Universitaria, 2003.

Nanni, Carlo. L'educazione tra crisi e ricerca di senso. Roma: Libreria Ateneo Salesiano, 1986.

Paolo VI. Evangelii Nuntiandi. Vatican: Libreria Editrice Vaticana, 1975.

Povilus, Judit. Gesù in mezzo nel pensiero di Chiara Lubich. Roma: Città Nuova, 1981. 
Quando la scuola educa, ed. Michele De Beni. Roma: Città Nuova, 2017.

Scilironi, Carlo. In cammino verso l'uomo. Milano: S. Paolo, 1994.

Suchodolski, Bodgan. Pedagogia dell'essenza e pedagogia dell'esistenza. Roma: Armando, 1965.

Zambrano, Maria. Chiari nel bosco. Milano: Feltrinelli, 1991. 
